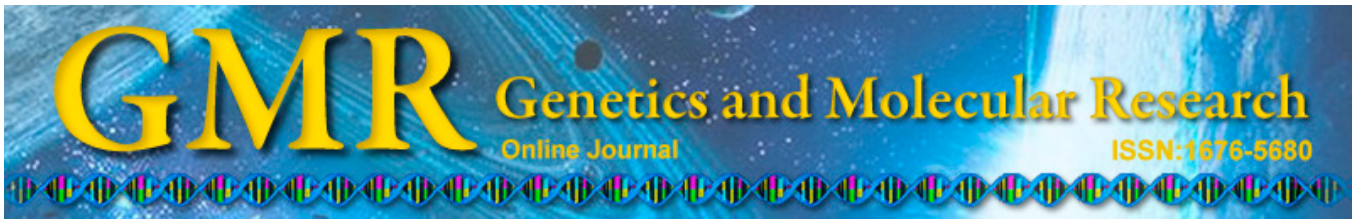

\title{
Apoptosis induced by lipid-associated membrane proteins from Mycoplasma hyopneumoniae in a porcine lung epithelial cell line with the involvement of caspase 3 and the MAPK pathway
}

\author{
B. Ni*, F.F. Bai*, Y. Wei, M.J. Liu, Z.X. Feng, Q.Y. Xiong, L.Z. Hua and \\ G.Q. Shao
}

Institute of Veterinary Medicine, Jiangsu Academy of Agricultural Sciences, Key Laboratory of Veterinary Biological Engineering and Technology, Ministry of Agriculture,

National Center for Engineering Research of Veterinary Bio-Products, Nanjing, China

*These authors contributed equally to this study.

Corresponding author: G.Q. Shao

E-mail: gqshaojaas@gmail.com

Genet. Mol. Res. 14 (3): 11429-11443 (2015)

Received January 15, 2015

Accepted May 15, 2015

Published September 25, 2015

DOI http://dx.doi.org/10.4238/2015.September.25.10

\begin{abstract}
Lipid-associated membrane proteins (LAMPs) are important in the pathogenicity of the Mycoplasma genus of bacteria. We investigated whether Mycoplasma hyopneumoniae LAMPs have pathogenic potential by inducing apoptosis in a St. Jude porcine lung epithelial cell line (SJPL). LAMPs from a pathogenic strain of M. hyopneumoniae (strain 232) were used in the research. Our investigation made use of diamidino-phenylindole (DAPI) and acridine orange/ethidium bromide (AO/EB) staining, terminal dexynucleotidyl transferase (TdT)-mediated dUTP nick end labeling (TUNEL) analysis,
\end{abstract}


and Annexin-V-propidium iodide staining. After LAMP treatment for $24 \mathrm{~h}$, typical changes were induced, chromosomes were concentrated, apoptotic bodies were observed, the 3'-OH groups of cleaved genomes were exposed, and the percentage of apoptotic cells reached $36.5 \pm$ $11.66 \%$. Caspase 3 and caspase 8 were activated and cytochrome $c$ (cyt $c$ ) was released from the mitochondria into the cytoplasm; poly ADP ribose polymerase (PARP) was digested into two fragments; $\mathrm{p} 38$ mitogen-activated protein kinase (MAPK) was phosphorylated; and the expression of pro-apoptosis protein Bax increased while the antiapoptosis protein Bcl-2 decreased. LAMPs also stimulated SJPL cells to produce nitric oxide (NO) and superoxide. This study demonstrated that LAMPs from $M$. hyopneumoniae can induce apoptosis in SJPL cells through the activation of caspase 3 , caspase 8 , cyt $c$, Bax, and $\mathrm{p} 38$ MAPK, thereby contributing to our understanding of the pathogenesis of $M$. hyopneumoniae, which should improve the treatment of $M$. hyopneumoniae infections.

Key words: Mycoplasma hyopneumoniae; Apoptosis; Caspase 3; Lipid-associated membrane proteins

\section{INTRODUCTION}

Mycoplasma hyopneumoniae is the causative agent of enzootic pneumonia, which is characterized by chronic non-productive coughing, poor growth rate, and a lower feed conversion ratio. Moreover, swine are more susceptible to other respiratory pathogens when infected with $M$. hyopneumoniae leading to porcine respiratory disease complex (PRDC) (Thacker and Minion, 2012). M. hyopneumoniae infection occurs worldwide and causes significant economic losses to the swine industry. Nevertheless, its mechanism of pathogenesis and its associated virulence factors remain unclear.

Research on the pathogenesis of $M$. hyopneumoniae has recently focused on genome and proteomics analyses, adherence, and inflammatory response. Adherence is the crucial first step in the interaction with the host. In addition to early detection of P97, an adhesion factor of M. hyopneumoniae (Zhang et al., 1995), Mhp684 (P146) (Bogema et al., 2012), and P116 (Seymour et al., 2010) are also important adhesion and virulence factors. After adherence to the respiratory epithelium cilia of the host, M. hyopneumoniae disrupts the mucociliary function and induces chronic inflammation. M. hyopneumoniae can induce nitrogen oxide (NO) and pro-inflammatory cytokines in MH-S and RAW 264.7 cells through nuclear factor kappa B (NF-kB) and mitogen-activated protein kinase (MAPK) pathways (Damte et al., 2011; Hwang et al., 2011). Inflammatory cytokines are associated with the development of M. hyopneumoniae infection and can contribute to disease severity. Although the adhesion mechanism is still unclear, damage to host cilia and ciliated epithelial cells is believed to play an important role in the pathogenicity of M. hyopneumoniae.

The Mycoplasma is bound by a single limiting cytoplasmic membrane with abundant lipoproteins known as lipid-associated membrane proteins (LAMPs) (You et al., 2006). Most of the LAMPs are exposed to the extracellular milieu and some have either been described as virulence factors (Szczepanek et al., 2010) or targets for antibodies (Washburn et al., 2003). 
Moreover, the membrane proteins of $M$. hyopneumoniae undergo incomplete proteolytic cleavage to create a highly variant and dynamic surface topography (Bogema et al., 2012). Many of these protein fragments interact with host extracellular matrix proteins to help maintain their association with the mycoplasma cell surface and to assist with adherence to host tissues (Deutscher et al., 2012).

Mycoplasma LAMPs play a critical role in the host immune response by mediating the activation of a variety of important pathways, MAPK, and NF- $\mathrm{KB}$ (Shimizu et al., 2008). For example, LAMPs from $M$. genitalium induce pro-inflammatory cytokines, such as tumor necrosis factor- $\alpha$ (TNF- $\alpha$ ), interleukin-1 (IL-1), and IL-6, in human monocytic cells (Wu et al., 2008). The pro-inflammatory cytokines are believed to play a prominent role in the symptoms of enzootic pneumonia, causing the characteristic accumulation of large numbers of leukocytes in the pulmonary tissues (Muneta et al., 2008). Recent evidence suggests that cell apoptosis contributes to the pathogenesis of a number of diseases, including many infectious and autoimmune diseases. Moreover, it is now known that Mycoplasma bacteria are capable of inducing apoptosis through toll-like receptors (TLRs) (Into et al., 2002, 2004; Into and Shibata, 2005). These studies support the idea that lipoprotein-induced apoptosis may significantly contribute to the pathogenesis of Mycoplasma bacteria.

There have been no reports as to whether M. hyopneumoniae LAMPs are capable of inducing apoptosis in the St. Jude porcine lung epithelial cell line (SJPL). This study was therefore designed to investigate whether M. hyopneumoniae LAMPs can induce apoptosis in SJPL cells, and the possible molecular mechanisms involved.

\section{MATERIAL AND METHODS}

\section{Mycoplasma culture and LAMPs fraction preparation}

M. hyopneumoniae (strain 232, ATCC 27714) was cultivated in $\mathrm{KM} 2$ media at $37^{\circ} \mathrm{C}$. The preparation of LAMPs followed the methods described previously (Rawadi and RomanRoman, 1996). The culture was pelleted by centrifugation and resuspended in $5 \mathrm{~mL}$ Tris-buffered saline $(50 \mathrm{mM}$ Tris, $\mathrm{pH} 8.0,0.15 \mathrm{M} \mathrm{NaCl})$ with $1 \mathrm{mM}$ ethylenediaminetetraacetic acid (TBSE). Triton X-114 was added to a final concentration of $2 \%$ and mixed, and the mixture was incubated at $4^{\circ} \mathrm{C}$ for $1 \mathrm{~h}$. The Triton X-114 lysate was incubated at $37^{\circ} \mathrm{C}$ for $10 \mathrm{~min}$ for phase separation and then centrifuged at $10,000 \mathrm{~g}$ for $20 \mathrm{~min}$. The upper aqueous phase was transferred to a second tube and replaced with an equal volume of TBSE. This phase separation was repeated twice. The final Triton X-114 phase was adjusted to the original volume with TBSE. Two-and-a-half volumes of ethanol were then added to precipitate membrane LAMPs overnight at $-20^{\circ} \mathrm{C}$. Precipitated materials were recovered by centrifugation and suspended in phosphate-buffered saline (PBS), and then lysed by sonication at $30 \mathrm{~Hz}$ in an ice bath using five 30-sec cycles with 1-min intervals between pulses. These preparations were incubated for $2 \mathrm{~h}$ with polymyxin $\mathrm{B}$ at $1000 \mathrm{U} / \mathrm{mL}$ prior to use. The protein concentration of LAMPs was measured using the bicinchoninic acid method (Beyotime, China).

\section{Cell culture and stimulation}

SJPL cells are an immortalized swine respiratory epithelial cell line and have been described as suitable for swine pathogen research (Seo et al., 2001). SJPL cells were cultured 
at $37^{\circ} \mathrm{C}$ in Dulbecco's modified Eagle's medium supplemented with $10 \%$ calf serum (Gibco, USA), $100 \mathrm{IU} / \mathrm{mL}$ penicillin, and $100 \mu \mathrm{g} / \mathrm{mL}$ streptomycin in a $5 \% \mathrm{CO}_{2}$ humidified air incubator. When grown to $70-80 \%$ confluence, cells were counted and cultured on 6 - or 96 -well plates.

\section{3-(4,5-dimethylthiazol-2-yl)-2,5-diphenyltetrazolium bromide (MTT) assay}

The effects of LAMPs on the viability of cells were determined by MTT assay, as described previously (Mosmann, 1983). There were $1 \times 10^{4}$ cells/well on a 96-well plate. After $24 \mathrm{~h}$ incubation, various concentrations of LAMPs from 0.1 to $0.6 \mathrm{mg} / \mathrm{mL}$ were added and the cells were further cultured for $48 \mathrm{~h}$. Thereafter, $20 \mu \mathrm{L}$ MTT $(5 \mu \mathrm{g} / \mathrm{mL})$ was added into each well after removal of the medium, and incubated for another $4 \mathrm{~h}$. The formazan crystals formed were dissolved with $150 \mu \mathrm{L}$ dimethyl sulfoxide. Absorbance was measured at $570 \mathrm{~nm}$ using a microplate reader (BioTek, USA). Assays were repeated at least three times.

Diamidino-phenylindole (DAPI), acridine orange/ethidium bromide (AO/EB), and terminal dexynucleotidyl transferase (TdT)-mediated dUTP nick end labeling (TUNEL) assays

DAPI staining solution (Beyotime, China) was used to detect the changes in nuclear morphology. Cells were seeded on 96-well plates treated with $0.2 \mathrm{mg} / \mathrm{mL}$ LAMPs for $24 \mathrm{~h}$, fixed with $4 \%$ paraformaldehyde, and washed with PBS three times. Subsequently, samples were stained with $35 \mu \mathrm{L}$ DAPI solution $(0.1 \mu \mathrm{g} / \mathrm{mL})$ for 3-5 min at room temperature in the dark. The morphology of cell nuclei was observed at a wavelength of $340 \mathrm{~nm}$ using a fluorescence microscope (Olympus, USA). AO/EB staining and exclusion dye methods were used to enable the differentiation between live and apoptotic cells. Cells were seeded on 96-well plates treated with $0.2 \mathrm{mg} / \mathrm{mL}$ LAMPs for $24 \mathrm{~h}$, fixed with $4 \%$ paraformaldehyde, and then washed with PBS three times. The AO/EB dye mix $(20 \mu \mathrm{L})$ (KeyGen, Normal/Apoptosis/ Necrotic Cell Detection Kit, China) was added to each well, and observed by fluorescence microscopy (Yang et al., 2013). The TUNEL assay was conducted using a One Step TUNEL Apoptosis Assay Kit according to the manufacturer instructions (In Situ Cell Death Detection Kit, Roche, USA). The TdT was added to every sample seeded on the 96-well plates for $1 \mathrm{~h}$ at $37^{\circ} \mathrm{C}$ and was then observed by fluorescence microscopy.

\section{Measurement of cell apoptosis}

Annexin-V-propidium iodide (PI) staining, which detects the exposure of phosphatidyl serine in early apoptosis, was carried out to enable the differentiation between live, early/late apoptotic, and dead cells. After 12-, 24-, and 36-h treatments with LAMPs, cells were collected and resuspended in $350 \mu \mathrm{L}$ binding buffer, while untreated cells were used as controls. Annexin-V and PI ( $3 \mu \mathrm{L}$ each) (BD, USA) were added to each sample, and mixed and incubated for $15 \mathrm{~min}$ at room temperature in the dark. The cell apoptosis assay was performed immediately on flow cytometry apparatus (BD, USA). Each experiment was performed at least three times.

\section{Caspase analysis}

The activities of caspase 3 and caspase 8 were measured using a Caspase 3 Activity 
Assay Kit and a Caspase 8 Activity Assay Kit, respectively (Beyotime, China), following the manufacturer instructions. Cells were collected after treatment with LAMPs at 1, 3, 6, 12, 24, and $36 \mathrm{~h}$. Prepared SJPL cells were washed with $4^{\circ} \mathrm{C}$ PBS buffer, and $100 \mu \mathrm{L}$ lysis buffer was added to the cells which were placed on ice for $15 \mathrm{~min}$, then centrifuged at $12,000 \mathrm{~g}$ for $15 \mathrm{~min}$. Reaction buffer $(80 \mu \mathrm{L})$ and $\mathrm{N}$-acetyl-Asp-Glu-Tyr-Glu-Asp-p-nitroanilide (AcDEVED- $p$ NA) $(10 \mu \mathrm{L})$ were added to $10 \mu \mathrm{L}$ of the resulting supernatant liquid. After incubating at $37^{\circ} \mathrm{C}$ for $2-3 \mathrm{~h}$ in darkness, the absorbance readings were measured at $405 \mathrm{~nm}$ using the lysis buffer and reaction buffer as blanks (Mosmann, 1983). Caspase 8 was detected in a similar way except that Ac-IETD- $p$ NA was used instead of Ac-DEVED- $p$ NA. Each experiment was carried out at least three times.

\section{Western blot analysis}

Cells were seeded at $2 \times 10^{5}$ cells/well on a 6-well plate. After $12 \mathrm{~h}$ of treatment as described above, cells were extracted using a lysis buffer (Beyotime, China). Untreated cells served as controls. The total protein concentration was measured and adjusted to $50 \mu \mathrm{g}$ for every sample. The protein was separated by $12 \%$ sodium dodecyl sulfate polyacrylamide gel electrophoresis (SDS-PAGE) and transferred to a nitrocellulose membrane. The membranes were blocked with 5\% bovine serum albumin in Tris-buffered saline containing Tween-20 (TBST) for $2 \mathrm{~h}$, incubated with primary antibody overnight at $4^{\circ} \mathrm{C}$, and then with horseradish peroxidaselabeled goat anti-mouse or horseradish peroxidase-labeled goat anti-rabbit antiserum (Boster, China) diluted 1:1000. The proteins were visualized using the ECL detection system (Bio-Rad, Molecular Imager, USA). Rabbit monoclonal antibodies against poly ADP ribose polymerase (PARP) and Bcl-2 and mouse monoclonal antibodies against $\beta$-actin, Bax, and p38 MAPK were included in the experiments as primary antibody at dilutions of 1:1000 (Beyotime, China).

\section{Immunofluorescence staining of cyt $c$ analysis}

Cells seeded at $1 \times 10^{4}$ cells/well on a 96-well plate were stimulated with LAMPs for $12 \mathrm{~h}$ and then fixed with 4\% paraformaldehyde. The cells were later incubated with mouse monoclonal antibodies against cyt $c$ (Beyotime, China) and fluorescein isothiocyanate-labeled goat anti-mouse IgG antiserum (Boster, China) diluted at 1:1000. The release of cyt $c$ was observed under a fluorescence microscope.

\section{NO assay}

Cells were seeded at $1 \times 10^{4}$ cells/well on 96-well plates and then treated with LAMPs for $6,12,18$, and $24 \mathrm{~h}$. Untreated cells served as controls. Cell-free culture medium was collected for investigation. Nitrite is a stable byproduct of NO, which can be measured in the culture supernatant. To measure the amount of nitrite produced, $50 \mu \mathrm{L}$ of the cell-free culture medium was placed on to the enzyme-linked immunosorbent assay plate and $50 \mu \mathrm{L}$ of each Griess reagent I and II of the Nitric Oxide Assay Kit (Beyotime, China) was added to each well, all at room temperature and protected from light. Optical density (OD) was measured at $570 \mathrm{~nm}$, using a BioTek micro plate reader. The concentration of nitrite in each sample was calculated using a standard curve generated using sodium nitrite. Each experiment was carried out at least three times. 


\section{Superoxide assay}

Superoxide is able to reduce the water-soluble tetrazolium salt of MTT-1 (WST-1) to a soluble colored material allowing detection. Superoxide anion levels were measured using a superoxide assay kit (Beyotime, China) following the manufacturer instructions. Briefly, cells were seeded at $1 \times 10^{4}$ cells/well into a 96-well plate. After washing with PBS, the cells were incubated with $200 \mu \mathrm{L}$ superoxide assay solution including WST- 1 and catalase at $37^{\circ} \mathrm{C}$ for 3 $\mathrm{min}$. The LAMPs were added while ensuring that blank and negative controls were also set up. Stimulation was carried out at time intervals of $0.5,1,2,4$, and $8 \mathrm{~h}$, after which absorbance of the plates was read at $450 \mathrm{~nm}$. The assays were repeated at least three times.

\section{Statistics}

All the experiments were performed in their entirety at least three times. Results are reported as means \pm standard deviation and were compared using a one-way analysis of variance. The differences between the groups were compared by using multiple comparisons (Bonferroni $t$-text). P values $<0.05$ were considered to be statistically significant.

\section{RESULTS}

\section{M. hyopneumoniae LAMPs had a cytotoxic effect on SJPL cells}

The MTT assay was used to measure the cytotoxicity induced by LAMPs on SJPL cells. The average inhibition rates were $33.74 \%$ for a concentration of $0.1 \mathrm{mg} / \mathrm{mL}, 51.14 \%$ for $0.2 \mathrm{mg} / \mathrm{mL}, 65.68 \%$ for $0.3 \mathrm{mg} / \mathrm{mL}$, and $97.52 \%$ for $0.4 \mathrm{mg} / \mathrm{mL}$ after $48 \mathrm{~h}$ of treatment. However, when the concentration of LAMPs increased from 0.4 to $0.6 \mathrm{mg} / \mathrm{mL}$, the level of cytotoxicity decreased (Figure 1). The inhibitory concentration $50\left(\mathrm{IC}_{50}\right)$ of $0.2 \mathrm{mg} / \mathrm{mL}$ was chosen to be the concentration for inducing apoptosis and this concentration was used in the subsequent experiments.

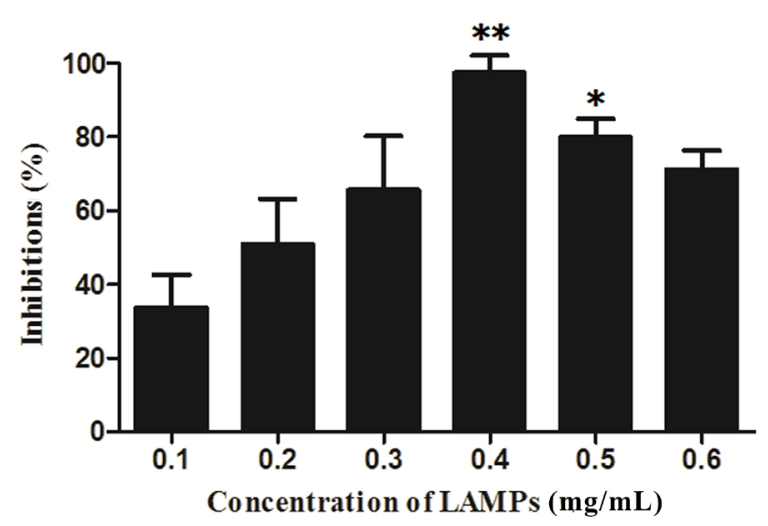

Figure 1. St. Jude porcine lung epithelial cell line (SJPL) cell viability following Mycoplasma hyopneumoniae lipid-associated membrane protein (LAMP) treatment. Viability (\%) was measured by the 3-(4,5-dimethylthiazol2-yl)-2,5-diphenyltetrazolium bromide (MTT) assay at different concentration intervals from 0.1 to $0.6 \mathrm{mg} / \mathrm{mL}$ of LAMPs for $48 \mathrm{~h}$. $* \mathrm{P}<0.05$ and $* * \mathrm{P}<0.01$ compared with untreated cells. 


\section{M. hyopneumoniae LAMPs induced apoptotic body formation, nuclear condensation, and TUNEL-positive cells}

To determine whether the cytotoxic activities of LAMPs were related to the induction of apoptosis, the morphological changes of nuclear and DNA fragmentation of SJPL cells were investigated using DAPI and AO/EB staining, while the TUNEL assay was used to confirm apoptosis. A series of typical lesions related to apoptosis could be observed after treatment with LAMPs for $24 \mathrm{~h}$ in SJPL cells. First, the nuclear morphology had changed. Untreated control cells showed clear-edged, uniformly stained, blue nuclei. After treatment with LAMPs for 24 $\mathrm{h}$, both nuclear condensation and karyorrhexis, which are characteristic of apoptosis, were observed in the treated SJPL cells. The nuclei had crumpled into irregular shapes, and had bright blue chromatid conglomerations and apoptotic bodies (Figure 2A). Owing to the genomic breakage, exposed 3'-OH groups could be labeled with dUTP-fluorescein isothiocyanate to show green fluorescence. As shown in Figure 2B, there were few TUNEL-positive cells in the control group, while the LAMPs-stimulated group had many more TUNEL-positive cells. AO stained the nuclear membrane emitting green fluorescence, while EB only stained cells that had lost membrane integrity (Orrenius et al., 2007). The control group containing mostly live cells showed green nuclei, whereas cells in the LAMPs-stimulated group were apoptotic and the nuclei were orange (Figure 2C).
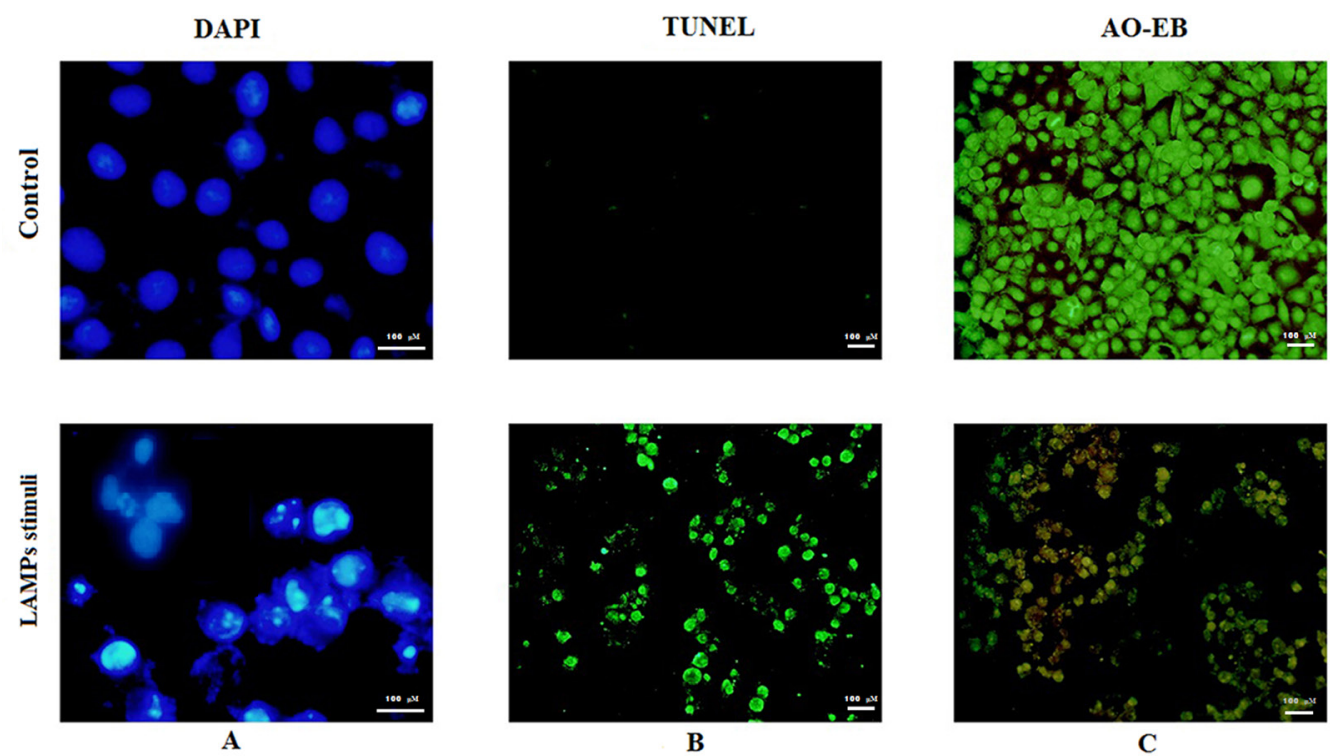

Figure 2. Apoptotic features induced by lipid-associated membrane proteins (LAMPs) in St. Jude porcine lung epithelial cell line (SJPL) cells. Stimulated groups were treated with $0.2 \mathrm{mg} / \mathrm{mL} \mathrm{LAMPs}$ for $24 \mathrm{~h}$ while untreated cells served as controls. (A) Representative images show nuclear morphology as detected by diamidinophenylindole (DAPI) staining (blue) under fluorescence microscopy. (B) Representative images of terminal dexynucleotidyltransferase (TdT)-mediated dUTP nick end labeling (TUNEL) staining showing that LAMPs induced more apoptotic cells. (C) Representative images of acridine orange (AO)-stained cells (red channel) and ethidium bromide (EB)-stained cells (green channel) were merged to distinguish living and apoptotic cells. 


\section{M. hyopneumoniae LAMPs increased apoptotic rates in SJPL cells}

The apoptotic ratios induced by LAMPs were assessed by flow cytometry through Annexin-V-PI staining. In live cells, phosphatide serine is located on the cytoplasmic surface of the cell membrane. However, in apoptotic cells, phosphatidyl serine is translocated from the inner to the outer leaflet of the membrane, so that it was susceptible to Annexin-V staining. PI, a nucleic acid dye, could only permeate into late apoptotic and dead cells. Early and late apoptotic cells and dead cells could be distinguished using Annexin V-PI staining in combination. The results indicated that the percentages of apoptotic cells (Annexin- $\mathrm{V}^{+} \mathrm{PI}^{+}$and Annexin- $\left.\mathrm{V}^{+} \mathrm{PI}^{-}\right)$were $13.6 \pm 1.2,36.5 \pm 11.6$, and $34.1 \pm 13.1 \%$ after treatment with LAMPs for 12,24 , and $36 \mathrm{~h}$, respectively (Figure 3 ). From these results it was confirmed that LAMPs induced apoptosis in SJPL cells and that the maximum number of apoptotic cells occurred after $24 \mathrm{~h}$ of incubation.

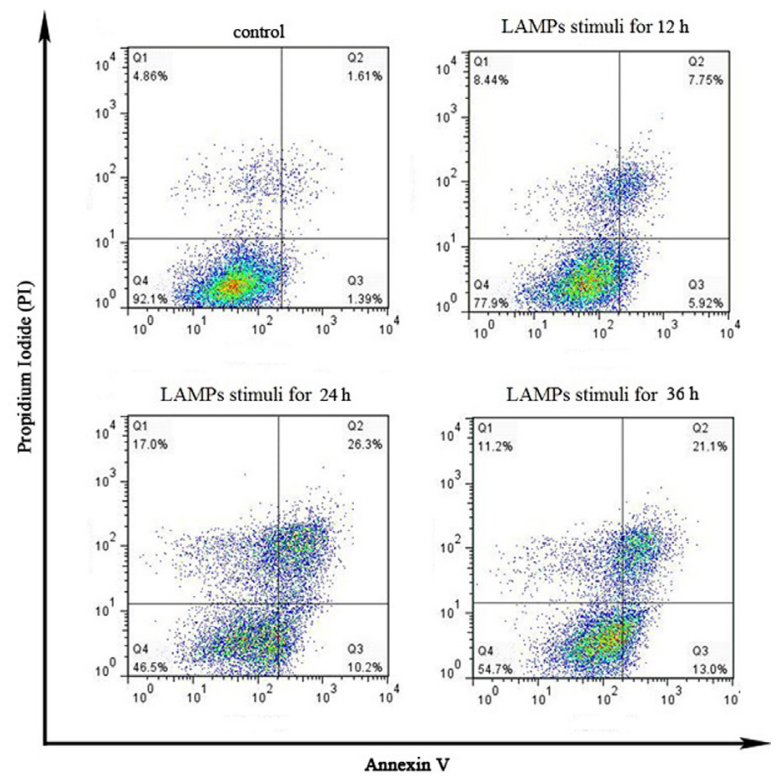

Figure 3. Apoptotic rates in St. Jude porcine lung epithelial cell line (SJPL) cells induced by lipid-associated membrane proteins (LAMPs). Cells were seeded on 6-well plates then stimulated with $0.2 \mathrm{mg} / \mathrm{mL}$ LAMPs for 12, 24 , and $36 \mathrm{~h}$, while untreated cells served as controls. Cells were stained with both Annexin-V and propidium iodide (PI). Apoptosis was measured by flow cytometry. Double-negative staining representing living cells is shown in quadrant Q4, positive staining for Annexin-V and negative staining for PI represent the early apoptotic stage shown in quadrant Q3, double-positive staining representing the late apoptotic stage is shown in quadrant Q2, and dead cells are concentrated in quadrant Q1.

\section{M. hyopneumoniae LAMPs activated caspase 3 and caspase 8 in SJPL cells}

The caspase protein family is involved in cell growth, differentiation, and apoptosis, and caspase 3 and caspase 8 play a central role in apoptosis. To explore the mechanism of LAMPs-induced apoptosis in SJPL cells, the activity of caspase 3 and caspase 8 was examined. The results indicated that caspase 3 and caspase 8 had been activated in the apoptotic 
pathway. After treatment with LAMPs for $6 \mathrm{~h}$, the maximum enzyme activity of caspase 3 was increased 40 -fold compared with the untreated cells, while the maximum enzyme activity of caspase 8 was a 4 -fold increase compared with the untreated cells (Figure 4A). However, as the time increased from 6 to $36 \mathrm{~h}$, the level of enzyme activity decreased.
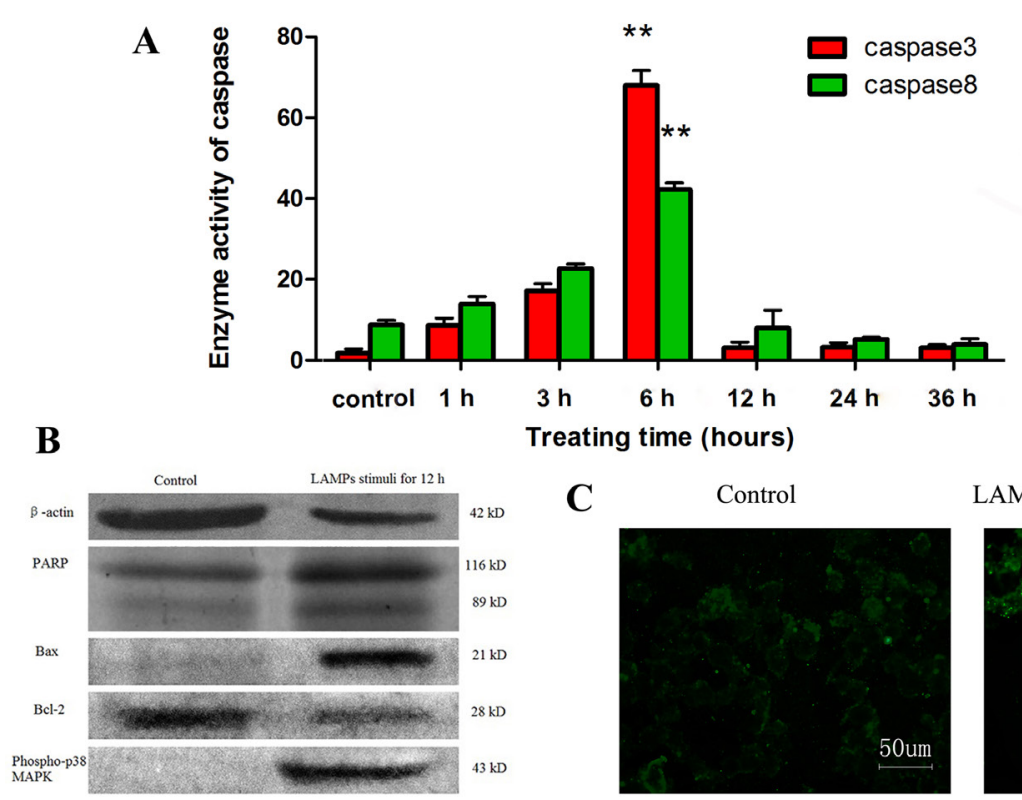

LAMPs stimuli for 12 hours

Figure 4. (A) Activation of caspase 3 and caspase 8 by Mycoplasma hyopneumoniae lipid-associated membrane proteins (LAMPs). Cells were treated or untreated with $0.2 \mathrm{mg} / \mathrm{mL}$ LAMPs for 1, 3, 6, 12, 24, and $36 \mathrm{~h}$. Caspase activation was indicated by Beyotime kits according to manufacturer instructions. Data are reported as means \pm standard deviation of three replicates. $* \mathrm{P}<0.05$ and $* * \mathrm{P}<0.01$ compared with untreated cells. (B) The changes of signaling proteins after treatment with LAMPs for $12 \mathrm{~h}$, including PARP, Bax, Bcl-2, p38 MAPK, and $\beta$-actin as loading control. (C) The release of cyt $c$ after stimulation with $0.2 \mathrm{mg} / \mathrm{mL}$ LAMPs for $24 \mathrm{~h}$. Images were acquired by fluorescence microscopy.

\section{M. hyopneumoniae LAMPs effects on PARP, p38 MAPK, Bax, Bcl-2, and cyt $c$}

After treatment with LAMPs, some proteins related to the apoptotic pathway changed. PARP was cut into two fragments, p38 MAPK was phosphorylated, the expression of proapoptosis protein Bax increased, while the expression of anti-apoptosis protein $\mathrm{Bcl}-2$ decreased after treatment with LAMPs for $12 \mathrm{~h}$. $\beta$-actin was used as a loading control (Figure 4B). Cyt $c$ was released from the mitochondria to the cytoplasm, and was detected using a specific antibody showing fluorescence in the cytoplasm (Figure 4C).

\section{Induction of NO and reactive oxygen species (ROS) by LAMPs in SJPL cells}

Since high concentrations of NO and peroxidation damage caused by reactive oxygen species (ROS) can promote apoptosis, NO and superoxide were examined to confirm apoptosis induced by LAMPs. The production of $\mathrm{NO}$ had a positive correlation with the interaction time from 6 to $12 \mathrm{~h}$ (Figure 5A). The maximum concentration of NO was 2-fold higher com- 
pared with the untreated cells at $12 \mathrm{~h}$. However, when the interaction time increased from 12 to $24 \mathrm{~h}$, the level of NO decreased. Moreover, LAMPs induced production of ROS superoxide, and the concentration was a 1.5-, 3-, 2.5-, 1.8-, and 1.9-fold increased after treatment with LAMPs for $0.5,1,2,4$, and $8 \mathrm{~h}$, respectively, when compared with the untreated cells (Figure 5B). LAMPs can neither react with the Griess reagent nor have the ability to reduce WST-1.
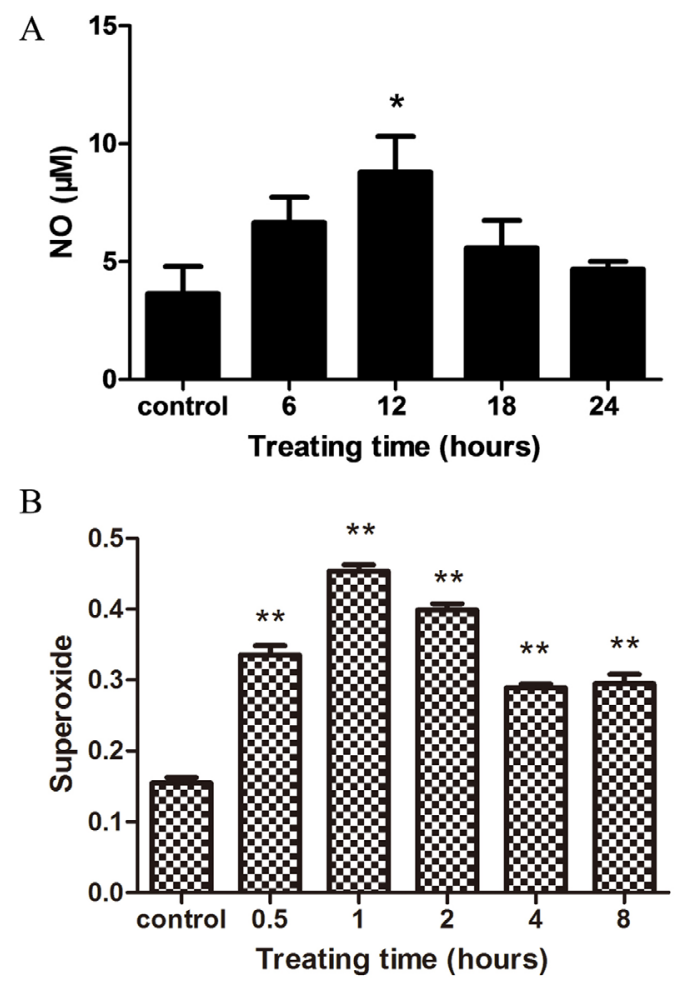

Figure 5. Effects of lipid-associated membrane proteins (LAMPs) on nitric oxide (NO) and superoxide production in St. Jude porcine lung epithelial cell line (SJPL) cells. (A) Cells were treated with LAMPs at different time intervals from 6 to $24 \mathrm{~h}$. (B) Cells were treated with $0.2 \mathrm{mg} / \mathrm{mL}$ LAMPs for $0.5,1,2$, 4, and $8 \mathrm{~h}$ for measuring superoxide levels. $* \mathrm{P}<0.05$ and $* * \mathrm{P}<0.01$ compared with untreated cells.

\section{DISCUSSION}

LAMPs appear to play a central role in the development of Mycoplasma-associated diseases. One of the best documented effects of LAMPs on cells is the induction of apoptosis. In a previous study, we found that LAMPs of M. hyopneumoniae induced apoptosis in the porcine alveolar macrophage 3D4/21 cell line via NO production, superoxide anion production, and caspase-3 activation (Bai et al., 2013).

As previously reported, the pathogenesis of $M$. hyopneumoniae consists of two separate methods of causing disease including modulating the host immune response tract and causing physical damage to the respiratory epithelial cells (Wu et al., 2008). In this study, we used the SJPL to detect the injury induced by LAMPs in the epithelial cells. The results obtained from this study demonstrated that LAMPs from $M$. hyopneumoniae induced apop- 
tosis in SJPL cells. Importantly, this study has established that mitochondrial cyt $c$ release and caspase 3 activation are associated with apoptosis. In addition, the increase of ROS and NO production suggests that ROS and NO may trigger apoptosis. In summary, LAMPs could stimulate SJPL cells to produce ROS and NO, and induce apoptosis in SJPL cells with the activation of caspase 3, caspase 8, Bax, p38 MAPK, and the release of cyt $c$.

These studies confirmed the interaction between LAMPs and SJPL cells. Our results demonstrated that LAMPs have a cytotoxic effect on SJPL cells. Apoptosis is a physiological pattern of cell death characterized by a series of morphological features and apoptotic bodies. These changes reflect complex biochemical events carried out by a family of cysteine proteases called caspases (Favaloro et al., 2012). During the development of infectious diseases, apoptosis appears to be one of several host defense mechanisms against microbes (Behar et al., 2011). Moreover, in vivo experiments have also proven that apoptosis contributes to the host's defense against bacterial infection (Grassmé et al., 2001). However, excessive apoptosis is detrimental to the host, causing tissue damage.

Some Mycoplasma proteins have already been shown to induce apoptosis in various cell lines. As previously reported, M. penetrans LAMPs induce apoptosis in mouse macrophages through the activation of NF-kB (Zeng et al., 2008). Moreover, M. genitalium LAMPs could stimulate THP- 1 cells to produce TNF- $\alpha$, IL- $1 \beta$, and IL- 6 in a dose- and time-dependent manner, and induce apoptosis by activating NF- $\mathrm{B}$ (Qiu et al., 2007). In addition, the lipidassociated molecules of $M$. fermentans induce death in the myelomonocytic cell lines U937 and HL-60 (Rawadi et al., 1996). A recombinant P48 protein of $M$. fermentans when expressed in $E$. coli as the maltose-binding protein fusion $\mathrm{rP} 48-\mathrm{MBP}$ was reported to induce apoptosis (Hall et al., 2000). In the present study, apoptotic bodies and TUNEL-positive cells appeared in the SJPL cells after LAMP treatment, as detected by DAPI, AO/EB staining, and TUNEL assays. The possible molecular mechanisms responsible for the induction of apoptosis were investigated by characterizing the activation of caspases.

Caspase 3 plays a central role in the execution of apoptosis and is also an important component of the cytotoxicity mechanism of cytotoxic T lymphocytes (MacKenzie and Clark, 2012). Activated caspase 3 may induce apoptosis through PARP digestion and possibly through digestion of other signaling enzymes or proteins of the pathway. PARP is one of the main proteolytic substrates of caspase 3, which is generally involved in DNA repair and safeguarding genetic integrity. At the beginning of apoptosis, the 116-kDa PARP protein is inactivated by cleavage into 24 - and $89-\mathrm{kDa}$ fragments by caspase 3 , leaving it unable to effect the repair of damaged DNA. The activity of $\mathrm{Ca}^{2+} / \mathrm{Mg}^{2+}$-dependent endonuclease DNAS1L3 increases, and it is regulated by PARP; this causes increased cleavage of chromosomal DNA leading to apoptosis (Janssens and Tinel, 2012). Caspase 8 also plays an important role in apoptosis. As the first among the caspase family of proteins to be activated, it then activates caspase 3 so that apoptosis can proceed (Beaudouin et al., 2013). Our results indicate that both caspase 3 and caspase 8 were activated after LAMPs treatment.

p38 is a central control point of the MAPK cascade in the regulation of intracellular signal transduction systems. Through phosphorylation, p38 MAPK mediates growth, development, differentiation, and death of cells (Deutscher et al., 2012). p38 MAPK plays a central role in refractory ceramic fibers-induced apoptosis in BEAS-2B cells. Exposure of BEAS-2B cells and positive control groups resulted in significantly increased expression of phosphop38 MAPK and caspase 3 compared with the normal group (Zhang et al., 2013). In sonodynamic therapy-induced U937 cell damage, the participation of MAPK signals was particularly 
examined; while the level of ROS increased, caspase 3 activation and DNA fragmentation emerged (Su et al., 2013). Moreover, MAPKs can also affect the regulation of ROS in cell death. $\mathrm{H}_{2} \mathrm{O}_{2}$ also has cytotoxic effects on HeLa cells, which can be enhanced by MAPK inhibitors. In particular, p38 inhibitors can increase ROS levels in $\mathrm{H}_{2} \mathrm{O}_{2}$-treated HeLa cells (Park, 2013). p38 MAPK plays an important role in NO-mediated cell death in neurons by stimulating Bax translocation to the mitochondria, activating the cell death pathway. Treatment with SB203580, a p38 MAPK inhibitor, diminishes caspase induction and protects SH-SY5Y cells and primary cultures of cortical neurons from NO-induced cell death (Ghatan et al., 2000). Overall, p38 MAPK may have played an important role in the regulation of apoptosis induced by LAMPs in this study and may be related to NO- and ROS-induced apoptosis.

Superoxide anions, hydrogen peroxide, and superoxides are ROS that can induce apoptosis through an oxidative stress response (Orrenius et al., 2007). As an important signaling molecule, NO plays an important role in the development of host immune system diseases (Zeng et al., 2008). In addition, both ROS and NO are capable of promoting apoptosis (Watson, et al., 1996; Vieira and Kroemer, 2003), so we hypothesized that ROS and NO might play a driving role in apoptosis induced by LAMPs in SJPL cells. Our results showed that LAMPs could trigger SJPL cells to produce NO and superoxide in a time-dependent manner. However, the concentrations peaked and then decreased over time. This may be explained by assuming that excessively extended periods of interaction time may be detrimental to SJPL cells, and thus, the production of $\mathrm{NO}$ and superoxide decreased.

Based on our data, as well as a large number of reported findings, we propose the following model for mycoplasma LAMPs-induced apoptosis in SJPL cells (Figure 6).

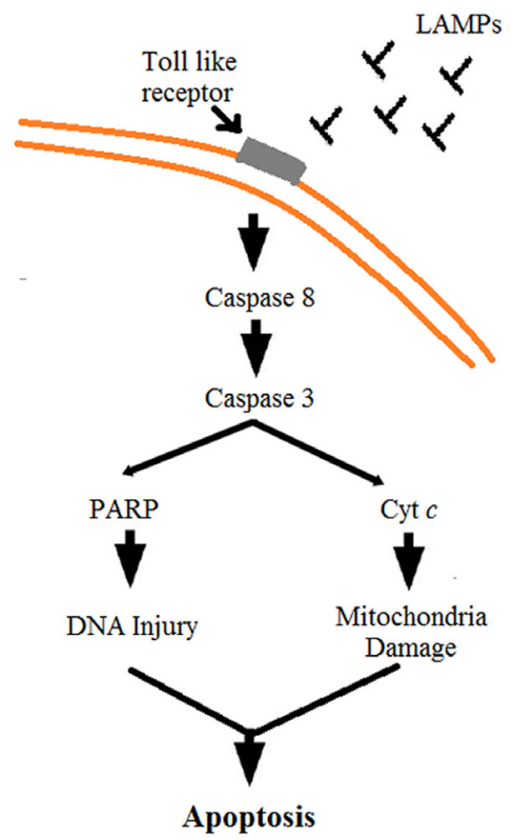

Figure 6. Model of lipid-associated membrane protein (LAMP)-induced apoptosis. Based on our experimental data and other reported research, we propose the following pathway for LAMP-induced apoptosis of St. Jude porcine lung epithelial cell line (SJPL) cells. 
Toll-like receptors are expressed on the surface of host cells and are responsible for the interaction with M. genitalium LAMPs (He et al., 2009). In our model, caspase 8 is first activated among the caspase family after which it promotes the downstream activation of caspase 3. Caspase 3 then digests PARP resulting in its loss of function and the cleavage of chromosomal DNA by the action of DNAS1L3. Furthermore, the damage to the mitochondria arising from the release of cytochrome $c$ from the mitochondria into the cytoplasm would also lead to apoptosis. Both DNA injury and mitochondrial damage are significant factors during the initiation of apoptosis. Our observations suggest that caspase 3 might function as an important intermediary in the apoptotic pathway induced by LAMPs in SJPL cells.

Successful pathogenic microorganisms that cause chronic infections avoid detection by the host immune mechanisms to promote long-term survival. One mechanism of immune evasion is the triggering of apoptosis in host cells, thereby escaping immune surveillance favoring their survival and propagation. The mechanisms of the pathogenesis of M. hyopneumoniae are complex and remain unclear. The chronic infection caused by M. hyopneumoniae proves that interactions between the host and M. hyopneumoniae are much more complex than expected. As the main component of the membrane, LAMPs constitute an important element in the interactions with the host. Moreover, the ability to induced apoptosis through LAMPhost cell interactions is probably a significant factor in the pathogenesis of M. hyopneumoniae. Studies on LAMPs may help to clarify the pathogenesis of M. hyopneumoniae.

This study demonstrated that LAMPs from M. hyopneumoniae have the ability to induce apoptosis in SJPL cells by the activation the pathway involving caspase 3, caspase 8, PARP, Bax, cyt $c$, and p38 MAPK. However, the complete signaling pathway of apoptosis still requires further exploration. The outcome of this study could improve our understanding of the pathogenesis of $M$. hyopneumoniae, while a better understanding of apoptotic pathway induction may help identify targets for treating $M$. hyopneumoniae infections.

\section{Conflicts of interest}

The authors declare no conflict of interest.

\section{ACKNOWLEDGMENTS}

Research supported by grants from the National Natural Science Foundation of China (\#31100135), the Jiangsu Agriculture Science and Technology Innovation Fund [\#CX (11) 4038], the Jiangsu Planned Projects for Postdoctoral Research Funds (\#1101159C), and the Natural Science Foundation of Jiangsu Province, China (\#BK20140754).

\section{REFERENCES}

Bai F, Ni B, Liu M, Feng Z, et al. (2013). Mycoplasma hyopneumoniae-derived lipid-associated membrane proteins induce apoptosis in porcine alveolar macrophage via increasing nitric oxide production, oxidative stress, and caspase-3 activation. Vet. Immunol. Immunopathol. 155: 155-161.

Beaudouin J, Liesche C, Aschenbrenner S, Hörner M, et al. (2013). Caspase-8 cleaves its substrates from the plasma membrane upon CD95-induced apoptosis. Cell Death Differ. 20: 599-610.

Behar SM, Martin CJ, Booty MG, Nishimura T, et al. (2011). Apoptosis is an innate defense function of macrophages against Mycobacterium tuberculosis. Mucosal Immunol. 4: 279-287.

Bogema DR, Deutscher AT, Woolley LK, Seymour LM, et al. (2012). Characterization of cleavage events in the 
multifunctional cilium adhesin Mhp684 (P146) reveals a mechanism by which Mycoplasma hyopneumoniae regulates surface topography. MBio. 3: e00282-11.

Damte D, Lee SJ, Hwang MH, Gebru E, et al. (2011). Inflammatory responses to Mycoplasma hyopneumoniae in murine alveolar macrophage cell lines. $N$ Z Vet. J. 59: 185-190.

Deutscher AT, Tacchi JL, Minion FC, Padula MP, et al. (2012). Mycoplasma hyopneumoniae Surface proteins Mhp385 and Mhp384 bind host cilia and glycosaminoglycans and are endoproteolytically processed by proteases that recognize different cleavage motifs. J. Proteome Res. 11: 1924-1936.

Favaloro B, Allocati N, Graziano V, Di Ilio C, et al. (2012). Role of apoptosis in disease. Aging (Albany NY). 4: 330-349.

Ghatan S, Larner S, Kinoshita Y, Hetman M, et al. (2000). p38 MAP kinase mediates bax translocation in nitric oxideinduced apoptosis in neurons. J. Cell Biol. 150: 335-347.

Grassmé H, Jendrossek V and Gulbins E (2001). Molecular mechanisms of bacteria induced apoptosis. Apoptosis. 6: 441-445.

Hall RE, Agarwal S and Kestler DP (2000). Induction of leukemia cell differentiation and apoptosis by recombinant P48, a modulin derived from Mycoplasma fermentans. Biochem. Biophys. Res. Commun. 269: 284-289.

He J, You X, Zeng Y, Yu M, et al. (2009). Mycoplasma genitalium-derived lipid-associated membrane proteins activate NF-kappaB through toll-like receptors 1, 2, and 6 and CD14 in a MyD88-dependent pathway. Clin. Vaccine Immunol. 16: $1750-1757$.

Hwang MH, Damte D, Lee JS, Gebru E, et al. (2011). Mycoplasma hyopneumoniae induces pro-inflammatory cytokine and nitric oxide production through NFkB and MAPK pathways in RAW264.7 cells. Vet. Res. Commun. 35: 21-34.

Into T and Shibata K (2005). Apoptosis signal-regulating kinase 1-mediated sustained p38 mitogen-activated protein kinase activation regulates mycoplasmal lipoprotein- and staphylococcal peptidoglycan-triggered Toll-like receptor 2 signalling pathways. Cell Microbiol. 7: 1305-1317.

Into T, Nodasaka Y, Hasebe A, Okuzawa T, et al. (2002). Mycoplasmal lipoproteins induce toll-like receptor 2- and caspases-mediated cell death in lymphocytes and monocytes. Microbiol. Immunol. 46: 265-276.

Into T, Kiura K, Yasuda M, Kataoka H, et al. (2004). Stimulation of human Toll-like receptor (TLR) 2 and TLR6 with membrane lipoproteins of Mycoplasma fermentans induces apoptotic cell death after NF-kappa B activation. Cell Microbiol. 6: 187-199.

Janssens S and Tinel A (2012). The PIDDosome, DNA-damage-induced apoptosis and beyond. Cell Death Differ. 19: 13-20.

MacKenzie SH and Clark AC (2012). Death by caspase dimerization. Adv. Exp. Med. Biol. 747: 55-73.

Mosmann T (1983). Rapid colorimetric assay for cellular growth and survival: application to proliferation and cytotoxicity assays. J. Immunol. Methods 65: 55-63.

Muneta Y, Minagawa Y, Shimoji Y, Ogawa Y, et al. (2008). Immune response of gnotobiotic piglets against Mycoplasma hyopneumoniae. J. Vet. Med. Sci. 70: 1065-1070.

Orrenius S, Gogvadze V and Zhivotovsky B (2007). Mitochondrial oxidative stress: implications for cell death. Annu. Rev. Pharmacol. Toxicol. 47: 143-183.

Park WH (2013). The effect of MAPK inhibitors and ROS modulators on cell growth and death of $\mathrm{H}_{2} \mathrm{O}_{2}$-treated HeLa cells. Mol. Med. Rep. 8: 557-564.

Qiu H, Wu YM, You XX, Yu MJ, et al. (2007). Mycoplasma genitalium lipid-associated membrane proteins induce human monocytic cell express proinflammatory cytokines and apoptosis by activating nuclear factor kappaB. Wei Sheng $W u$ Хие Bao. 47: 899-904.

Rawadi G and Roman-Roman S (1996). Mycoplasma membrane lipoproteins induced proinflammatory cytokines by a mechanism distinct from that of lipopolysaccharide. Infect. Immun. 64: 637-643.

Rawadi G, Roman-Roman S, Castedo M, Dutilleul V, et al. (1996). Effects of Mycoplasma fermentans on the myelomonocytic lineage. Different molecular entities with cytokine-inducing and cytocidal potential. J. Immunol. 156: 670-678.

Seo SH, Goloubeva O, Webby R and Webster RG (2001). Characterization of a porcine lung epithelial cell line suitable for influenza virus studies. J. Virol. 75: 9517-9525.

Seymour LM, Deutscher AT, Jenkins C, Kuit TA, et al. (2010). A processed multidomain Mycoplasma hyopneumoniae adhesin binds fibronectin, plasminogen, and swine respiratory cilia. J. Biol. Chem. 285: 33971-33978.

Shimizu T, Kida Y and Kuwano K (2008). A triacylated lipoprotein from Mycoplasma genitalium activates NF-kappaB through Toll-like receptor 1 (TLR1) and TLR2. Infect. Immun. 76: 3672-3678.

Su X, Wang P, Wang X, Guo L, et al. (2013). Involvement of MAPK activation and ROS generation in human leukemia U937 cells undergoing apoptosis in response to sonodynamic therapy. Int. J. Radiat. Biol. 89: 915-927.

Szczepanek SM, Frasca S Jr, Schumacher VL, Liao X, et al. (2010). Identification of lipoprotein MslA as a neoteric virulence factor of Mycoplasma gallisepticum. Infect. Immun. 78: 3475-3483. 
Thacker EL and Minion FC (2012). Mycoplasmosis. Diseases of Swine 10th. edn. 2012. John Wiley and Sons Inc., USA. 57: 779-797.

Vieira H and Kroemer G (2003). Mitochondria as targets of apoptosis regulation by nitric oxide. IUBMB Life. 55: 613-616.

Washburn LR, Bird DW and Dybvig K (2003). Restoration of cytoadherence to an adherence-deficient mutant of Mycoplasma arthritidis by genetic complementation. Infect. Immun. 71: 671-675.

Watson RW, Redmond HP, Wang JH and Bouchier-Hayes D (1996). Mechanisms involved in sodium arsenite-induced apoptosis of human neutrophils. J. Leukoc Biol. 60: 625-632.

Wu Y, Qiu H, Zeng Y, You X, et al. (2008). Mycoplasma genitalium lipoproteins induce human monocytic cell expression of proinflammatory cytokines and apoptosis by activating nuclear factor kappaB. Mediators Inflamm. 2008: 195427.

Yang S, Zhao Q, Xiang H, Liu M, et al. (2013). Antiproliferative activity and apoptosis-inducing mechanism of constituents from Toona sinensis on human cancer cells. Cancer Cell Int. 13: 12.

You XX, Zeng YH and Wu YM (2006). Interactions between mycoplasma lipid-associated membrane proteins and the host cells. J. Zhejiang Univ. Sci. B. 7: 342-350.

Zeng Y, Wu Y, Deng Z, You X, et al. (2008). Apoptosis induced by lipid-associated membrane proteins from Mycoplasma penetrans is mediated by nuclear factor kappaB activation in mouse macrophage. Can. J. Microbiol. 54: 150-158.

Zhang M, Zhu LJ, Xiao Y and Zhang X (2013). Effects of p38 mitogen-activated protein kinases on the apoptosis of human bronchial epithelial cells induced by refractory ceramic fibers in vitro. Zhonghua Lao Dong Wei Sheng Zhi Ye Bing Za Zhi. 31: 347-350.

Zhang Q, Young TF and Ross RF (1995). Identification and characterization of a Mycoplasma hyopneumoniae adhesin. Infect. Immun. 63: 1013-1019. 